UDC: 519.63

\title{
Full-wave 3D earthquake simulation using the double-couple model and the grid-characteristic method
}

\author{
R. A. Bagaeva , V. I. Golubev ${ }^{\text {b }}$ Yu. A. Golubeva ${ }^{c}$ \\ Moscow Institute of Physics and Technology, \\ 141700, Russia, Moscow Region, Dolgoprudny city, Institutskij lane, b. 9 \\ E-mail: 'ramazan.bagaev@phystech.edu, ${ }^{b}$ w.golubev@mail.ru, ${ }^{c}$ uma-mipt@mail.ru
}

Received 22.05.2019.

Accepted for publication 14.11.2019.

\begin{abstract}
One of the destroying natural processes is the initiation of the regional seismic activity. It leads to a large number of human deaths. Much effort has been made to develop precise and robust methods for the estimation of the seismic stability of buildings. One of the most common approaches is the natural frequency method. The obvious drawback of this approach is a low precision due to the model oversimplification. The other method is a detailed simulation of dynamic processes using the finite-element method. Unfortunately, the quality of simulations is not enough due to the difficulty of setting the correct free boundary condition. That is why the development of new numerical methods for seismic stability problems is a high priority nowadays.

The present work is devoted to the study of spatial dynamic processes occurring in geological medium during an earthquake. We describe a method for simulating seismic wave propagation from the hypocenter to the day surface. To describe physical processes, we use a system of partial differential equations for a linearly elastic body of the second order, which is solved numerically by a grid-characteristic method on parallelepiped meshes. The widely used geological hypocenter model, called the "double-couple" model, was incorporated into this numerical algorithm. In this case, any heterogeneities, such as geological layers with curvilinear boundaries, gas and fluid-filled cracks, fault planes, etc., may be explicitly taken into account.

In this paper, seismic waves emitted during the earthquake initiation process are numerically simulated. Two different models are used: the homogeneous half-space and the multilayered geological massif with the day surface. All of their parameters are set based on previously published scientific articles. The adequate coincidence of the simulation results is obtained. And discrepancies may be explained by differences in numerical methods used. The numerical approach described can be extended to more complex physical models of geological media.
\end{abstract}

Keywords: mathematical simulation, numerical method, grid-characteristic method, seismic waves, earthquake, seismic resistance

Citation: Computer Research and Modeling, 2019, vol. 11, no. 6, pp. 1061-1067 (Russian).

The reported study was funded by RFBR according to the research project No. 18-37-00127. 


\section{Introduction}

With the development of modern high performance computing systems scientists and engineers have got an opportunity to simulate complex applied problems in real scale. One of the destroying natural processes is the initiation of the regional seismic activity. The gradual accumulation of stresses in the geological massif is changed to a sharp slippage along the existing fault. This leads to a large number of human deaths. Considerable effort has gone into developing precise and robust methods for the estimation of the seismic stability of buildings.

One of the most common approaches is the natural frequency method [Fajfar, 2018]. The complex geometry of the building is simulated by the set of springs and masses. For this equivalent system all natural frequencies are calculated. And the seismic resistance estimation is based on the distance from characteristic frequencies of regional sources to the simulated spectrum. The obvious drawback of this approach is a low precision due to the model oversimplification. The other method is a detailed simulation of dynamic processes with the finite element method [Koketsu et al., 2004]. Recently it has been successfully combined with some of statistical estimations [Hariri-Ardebili et al., 2018]. The quality of simulations is not enough due to the difficulty of setting the correct free boundary condition.

It should be noted that wave processes must be described by a hyperbolic system of equations. The grid-characteristic method was developed for the numerical solution of this type of systems. Initially it was widely used for gas dynamic problems. Later it was successfully adopted for the dynamic elastic problem. It was used to simulate seismic responses from different geological structures [Golubev, Khokhlov, 2018]. Preliminary attempts with the simple source model were made to simulate earthquake problems [Beklemysheva et al., 2018].

In this report we present the results of application of the advanced source model [Aki, 1980] to the simulation of the seismic initiation process using the grid-characteristic method. The method proposed was successfully applied to multi-layered geological models. The results of numerical simulations were compared with data obtained by other authors. They are in a good agreement in general. Some discrepancies may be explained by differences in numerical methods used and some inaccuracy of calculation parameters in publications.

\section{Mathematical Model and Numerical Method}

The description of the dynamic behavior of the geological massif under the earthquake load in a far field is based on the system of equations of linear elasticity theory. It consists of the second Hooke's law and rheological relationships between stresses and strains. We have decided to highlight all heterogeneities explicitly, so the background model is homogeneous and isotropic, and these equations are valid:

$$
\begin{gathered}
\rho \frac{\partial v_{x}}{\partial t}=\frac{\partial \sigma_{x x}}{\partial x}+\frac{\partial \sigma_{x y}}{\partial y}+\frac{\partial \sigma_{x z}}{\partial z}, \\
\rho \frac{\partial v_{y}}{\partial t}=\frac{\partial \sigma_{y x}}{\partial x}+\frac{\partial \sigma_{y y}}{\partial y}+\frac{\partial \sigma_{y z}}{\partial z}, \\
\rho \frac{\partial v_{z}}{\partial t}=\frac{\partial \sigma_{z x}}{\partial x}+\frac{\partial \sigma_{z y}}{\partial y}+\frac{\partial \sigma_{z z}}{\partial z}, \\
\frac{\partial \sigma_{i j}}{\partial t}=\lambda \delta_{i j} \delta_{k l} \frac{\partial \varepsilon_{k l}}{\partial t}+\mu\left(\delta_{i k} \delta_{j l}+\delta_{i l} \delta_{j k}\right) \frac{\partial \varepsilon_{k l}}{\partial t},
\end{gathered}
$$

where $\sigma$ is the stress tensor, $\varepsilon$ is the strain tensor, $v_{i}$ is the component of the velocity vector, and $i$ and $j$ are equal to $\{x, y, z\}$. 
The procedure of the individual layer description was described and verified in [Golubev et al., 2017]. The general approach allows us to describe also geological fractures with arbitrary orientation and geometry [Golubev, Khokhlov, 2018]. To solve the governing system of equations, the gridcharacteristic method on structured meshes was used [Khokhlov, Golubev, 2019]. The system (1)-(4) can be rewritten in a different canonical form with the vector. $\vec{u}$ of unknowns

$$
\frac{\partial \vec{u}}{\partial t}+A_{x} \frac{\partial \vec{u}}{\partial x}+A_{y} \frac{\partial \vec{u}}{\partial y}+A_{z} \frac{\partial \vec{u}}{\partial z}=0 .
$$

After splitting the system (5) along coordinate directions the eigenvalues and eigenvectors can be found analytically. And along the characteristic curves these equations will be transformed as

$$
\begin{gathered}
\frac{\partial \vec{u}}{\partial t}+\Omega^{-1} \Lambda \Omega \frac{\partial \vec{u}}{\partial \xi}=0, \\
\frac{\partial \vec{q}}{\partial t}+\Lambda \frac{\partial \vec{q}}{\partial \xi}=0 .
\end{gathered}
$$

The system (7) is a set of transport equations with constant parameters. It is solved with $3^{\text {rd }}$ order in space in time using the Rusanov scheme. At the final stage, the vector $\vec{u}$ is calculated from the vector $\vec{q}$ multiplied by the matrix $\Omega^{-1}$. Due to the high computational complexity of the problem, parallel technologies MPI and OpenMP are intensively used.

One of the major questions is the mathematical model of the source. Previously the "slip along the fault" model was successfully used to describe dynamic processes in the 3D geological medium [Golubev, Golubeva, 2018]. In this model the crack plane exists for a long period of time. At some instant, due to an increase in regional stresses, the smallest movement occurs. One part of the massif moves with the constant vector $\vec{V}$, and the second part, with the opposite vector $\overrightarrow{-V}$. Three independent angles are necessary to specify the model uniquely (see Fig. 1, left). The magnitude of $\vec{V}$ can be estimated based on the day surface displacement map.
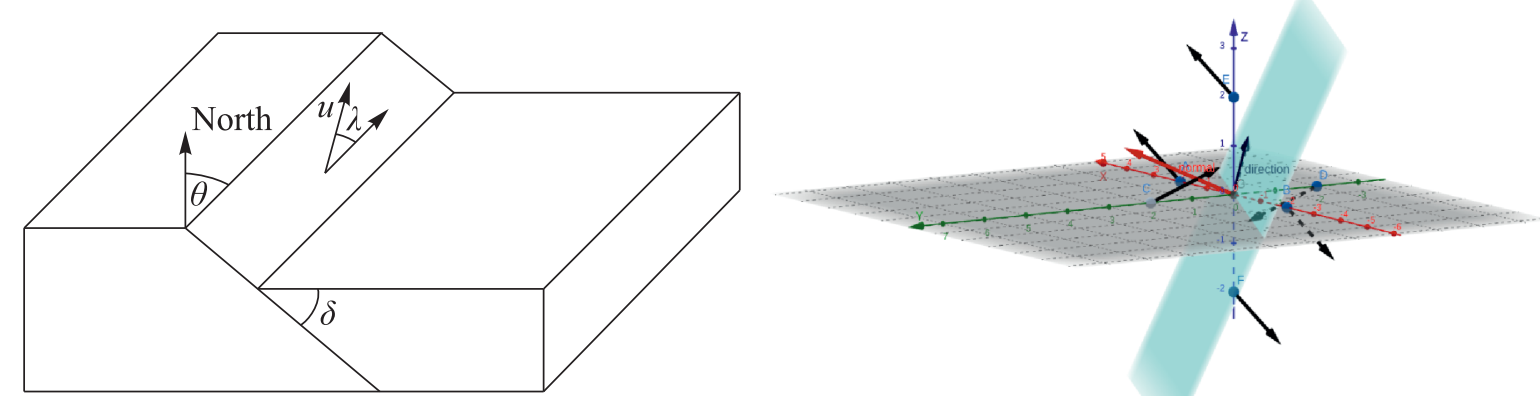

Fig. 1. The hypocenter model, called "the slip along the fault" (left) and "double-couple model" (right)

The drawback of this approach is the existence of many parameters (sizes of the region with the nonzero $\vec{V}$ vector) that cannot be easily estimated.

There is another widespread earthquake source model, the so-called "double-couple model" [Aki, 1980; Frankel, 1993]. The seismic moment tensor may be defined as

$$
M_{i j}(t)=M_{0}(t) *\left(n_{i} d_{j}+n_{j} d_{i}\right)
$$

where $\overrightarrow{\mathrm{n}}$ is the normal to the existing crack plane, $\overrightarrow{\mathrm{d}}$ is the displacement vector, $M_{0}(t)$ is the earthquake magnitude with the dependence on time. To set this source correctly inside our spatial mesh, we 
have to apply a set of force vectors in special nodes (see Fig. 1, right). If the hypocenter has coordinates $(i, j, k)$, then

$$
\begin{aligned}
& \vec{F}^{ \pm x}= \pm \frac{1}{2 h^{4}}\left(\begin{array}{c}
M_{x x} \\
M_{x y} \\
M_{x z}
\end{array}\right) \operatorname{in}(i \pm 1, j, k), \\
& \vec{F}^{ \pm y}= \pm \frac{1}{2 h^{4}}\left(\begin{array}{c}
M_{y x} \\
M_{y y} \\
M_{y z}
\end{array}\right) \operatorname{in}(i, j \pm 1, k), \\
& \vec{F}^{ \pm z}= \pm \frac{1}{2 h^{4}}\left(\begin{array}{c}
M_{z x} \\
M_{z y} \\
M_{z z}
\end{array}\right) \operatorname{in}(i, j, k \pm 1) .
\end{aligned}
$$

By construction, it is obvious that the sum of all forces is zero and the total moment vector is zero too. So, no constant shift or rotation is applied to the medium volume. The usage of the different $M_{0}(t)$ can influence the magnitude and the spectrum of the emitted seismic signal.

\section{Simulation Results}

Firstly, we have decided to validate the results of our numerical simulations with those obtained previously by Arthur Frankel for the 3D model of San Bernardino Valley [Frankel, 1993]. The relatively small size of the San Bernardino basin made it feasible to use a 3D simulation of frequencies of engineering significance. A 3D finite-difference scheme in terms of displacements was used. It was accurate to fourth order in space and to second order in time. An elastic attenuation was not included in the simulation, absorbing boundary conditions were applied on all sides of the grid, except for the free surface. The size of the domain was $37 \times 16 \times 7 \mathrm{~km}$. The source was placed at a depth of $6 \mathrm{~km}$, at the point $(20 \mathrm{~km}, 14 \mathrm{~km}, 1 \mathrm{~km})$. The "double-couple model" was used with the Gauss time dependence: $f(t)=e^{\frac{(t-1.5)^{2}}{0.25}}$. The fault orientation was $(0,-1,0)$ and the slip direction was $(1,0,0)$. All parameters were chosen for the M5 earthquake simulation. The parallelepiped mesh with spatial size $100 \mathrm{~m}$ was generated. The time step was $12 \mathrm{~ms}$ and a total of $48 \mathrm{~s}$ was simulated. Elastic parameters of layers are shown in Table 1 and are based on the paper [Frankel, 1993]. To compare the results, the $\mathrm{X}$-component of the velocity vector was measured at the point $(20 \mathrm{~km}, 6.3 \mathrm{~km})$ at the day surface. The model and source and receiver positions are depicted schematically in Fig. 2.
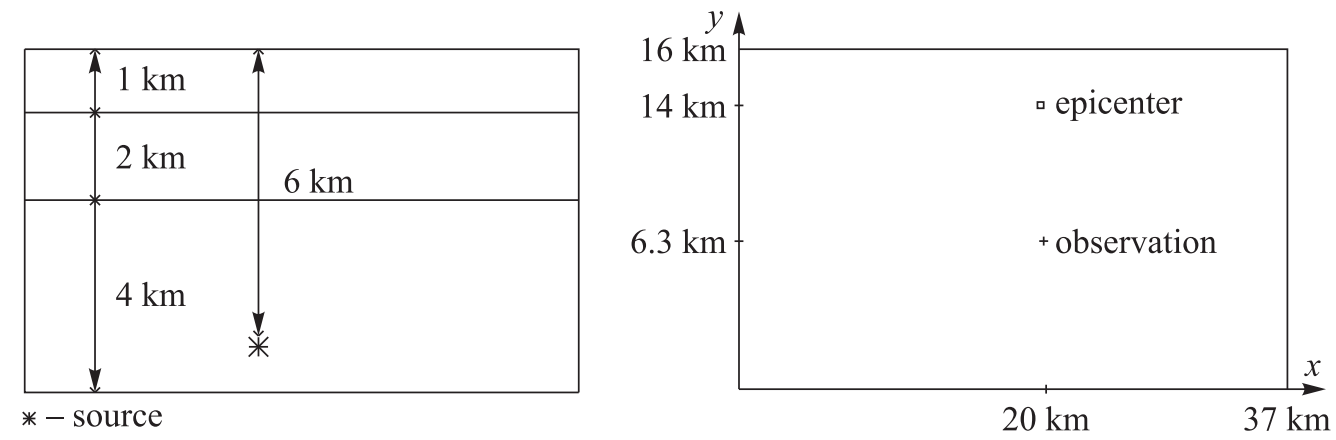

Fig. 2. Horizontal slice of the model (right) and the vertical slice of the model (left) based according to [Frankel, 1993] 
Table 1. Elastic parameters of geological layers

\begin{tabular}{|c|c|c|c|}
\hline Depth range, $\mathrm{km}$ & P-wave velocity, $\mathrm{km} / \mathrm{s}$ & S-wave velocity, $\mathrm{km} / \mathrm{s}$ & Density, $\mathrm{kg} / \mathrm{m}^{3}$ \\
\hline $0-1$ & 1.1 & 0.6 & 2000 \\
\hline $1-3$ & 3.5 & 2.0 & 2600 \\
\hline $3-7$ & 5.0 & 2.9 & 2600 \\
\hline
\end{tabular}

Figure 3 shows a comparison of our numerical simulation results with the data provided in [Frankel, 1993]. It is clearly seen that the dynamic and kinematic characteristics of first arrivals were reproduced well. Differences in the further signal may be due to the different orders of numerical schemes used, and some discrepancy was obviously introduced by the digitization of source/receiver positions from the figures placed in the reference paper.

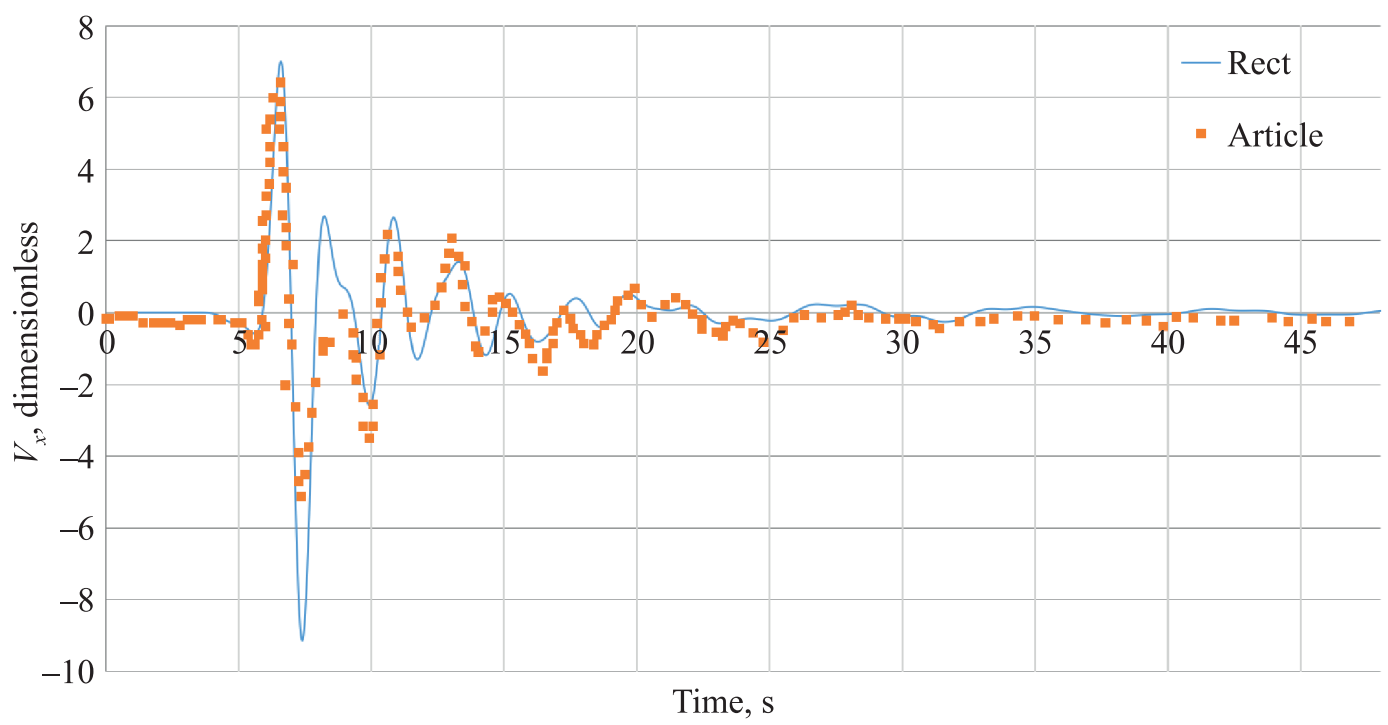

Fig. 3. Comparison of $V_{x}$ at the day surface at a distance of $7.7 \mathrm{~km}$ from the earthquake epicenter. Reference data were used from [Frankel, 1993]

Secondly, a reproduction of the computer experiment carried out by Robert W. Graves [Graves, 1996] using staggered-grid finite difference was performed. The single-layered model with $\mathrm{P}$-wave $4000 \mathrm{~m} / \mathrm{s}$, S-wave $2300 \mathrm{~m} / \mathrm{s}$ and density $1800 \mathrm{~kg} / \mathrm{m}^{3}$ was modeled. Its spatial size was $100 \times 100 \times 25 \mathrm{~km}$. The double-couple model with the magnitude $5 \mathrm{e}^{12}$, fault orientation $\left(0,-\frac{\sqrt{2}}{2}, \frac{\sqrt{2}}{2}\right)$ and the slip direction $\left(0, \frac{\sqrt{2}}{2}, \frac{\sqrt{2}}{2}\right)$ was investigated. The source spatial position was at the point $(50 \mathrm{~km}, 45 \mathrm{~km}, 2.5 \mathrm{~km})$. Time dependence was used as a triangle function with 45 degrees and duration $2 \mathrm{~s}$. The parallelepiped mesh with spatial size $250 \mathrm{~m}$ was generated. The time step was $25 \mathrm{~ms}$ and a total of $16 \mathrm{~s}$ was simulated. To compare the results, the Z-component of the velocity vector was measured at the point $(55 \mathrm{~km}, 50 \mathrm{~km})$ at the day surface.

Figure 5 shows a comparison of our numerical simulation results with the data provided in [Graves, 1996]. The main peak was reproduced well by time and amplitude too. Differences in the registered signals may be due to the different orders of numerical schemes used. It should be noted that the authors developed the calculation on the staggered grid, so their source contains not 6 , but 27 mesh nodes. 


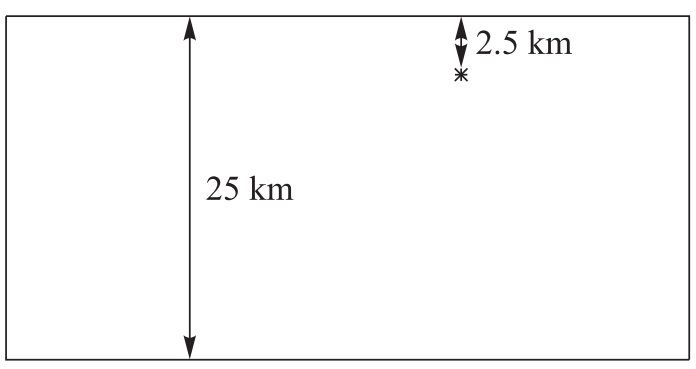

* - source

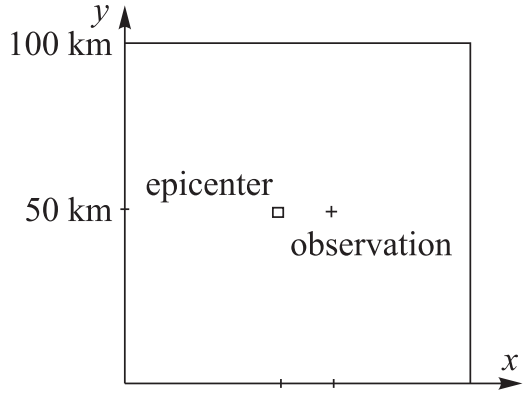

$45 \mathrm{~km} 55 \mathrm{~km} 100 \mathrm{~km}$

Fig. 4. The horizontal slice of the model (left) and the vertical slice of the model (right) based according to [Graves, 1996]

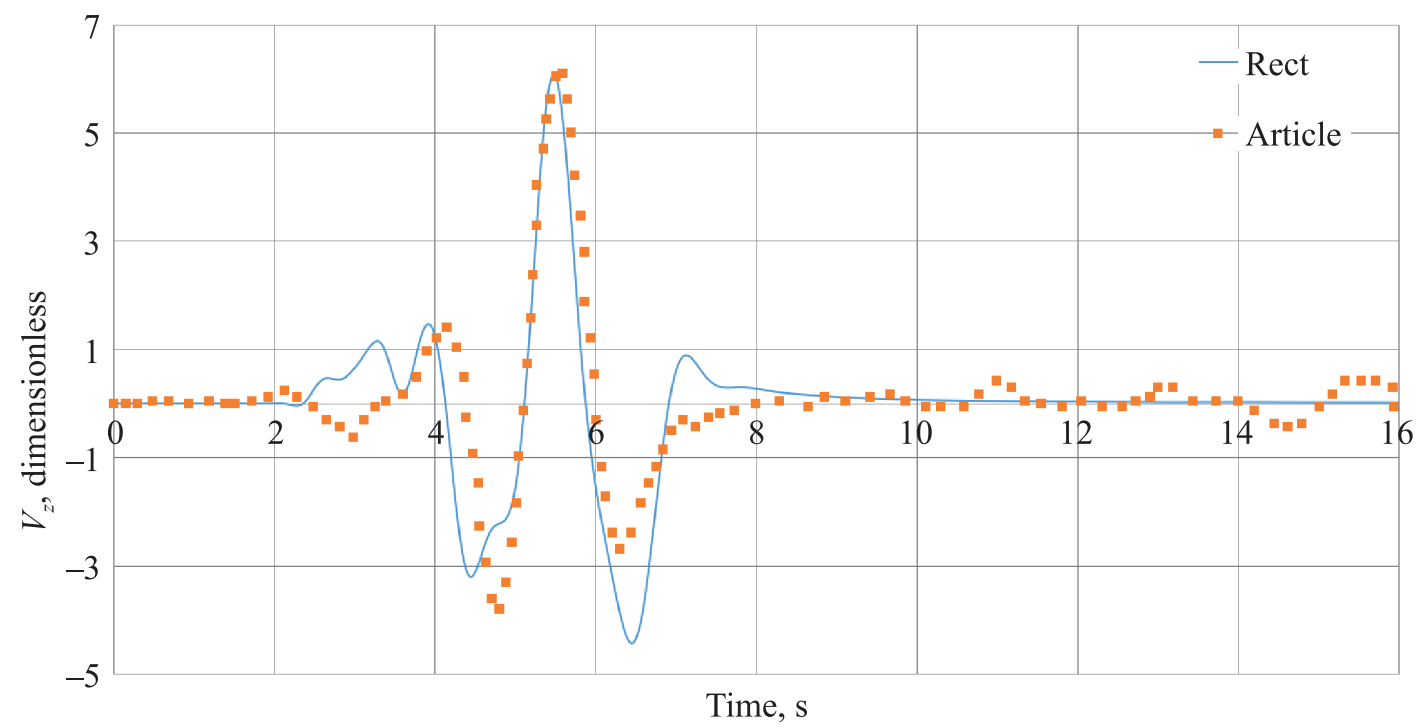

Fig. 5. Comparison of $\mathrm{V}_{\mathrm{z}}$. Reference data were used from [Graves, 1996]

\section{Conclusions}

This paper is concerned with the study of the problem of seismic wave propagation during the earthquake initiation process in the geological massif. The widely used geological model, called the "double-couple" model, was successfully combined with the numerical grid-characteristic method on parallelepiped meshes in the full-wave 3D case. This method was previously applied to simulate seismic responses from complex heterogeneous media: multilayered geological massifs, fractured gasfilled and fluid-filled regions, etc. Also, it was used to estimate the seismic resistance of ground facilities with the "slip along the fault" source model. The significant drawback of this approach is the inability to set correctly spatial sizes of the disturbed region. In this work, it was eliminated by taking into account a more reliable source model.

The proposed method was successfully applied to multilayered geological models. The results of numerical simulations were compared with data obtained by other authors. They are in a good agreement in general. Some discrepancies may be explained by differences in numerical methods used and some inaccuracy of calculation parameters in publications. Further research may be directed to supporting more complex physical models of geological media, for example, a poroelastic model. It may increase the reliability of simulations. 


\section{References}

Aki K., Richards P. G. Quantitative Seismology: Theory and Methods. - San Francisco: Freeman, 1980.

Beklemysheva K. A., Vasyukov A. V., Golubev V. I. et al. On the Estimation of Seismic Resistance of Modern Composite Oil Pipeline Elements // Dokl. Math. — 2018. — Vol. 97. — P. 184.

Fajfar P. Analysis in seismic provisions for buildings: past, present and future // Bull. Earthquake Eng. - 2018. - Vol. 16. - P. 2567.

Frankel A. Three-dimensional simulations of ground motions in the San Bernardino Valley, California, for hypothetical earthquakes on the San Andreas fault // Bulletin of the Seismological Society of America. - 1993. - Vol. 83, Is. 4. - P. 1020-1041.

Golubev V.I., Golubeva Yu. A. Full-wave simulation of the earthquake initiation process // CEUR Workshop Proceedings. - 2018.

Golubev V. I., Gilyazutdinov R. I., Petrov I. B., Khokhlov N. I., Vasyukov A. V. Simulation of dynamic processes in three-dimensional layered fractured media with the use of the grid-characteristic numerical method // Journal of Applied Mechanics and Technical Physics. - 2017. — Vol. 58, Is. 3. - P. 539-545.

Golubev V. I., Khokhlov N. I. Estimation of anisotropy of seismic response from fractured geological objects // Computer Research and Modeling. - 2018. - Vol. 10, Is. 2. - P. 231-240.

Graves $R$. W. Simulating seismic wave propagation in 3D elastic media using staggered-grid finite differences // Bulletin of the Seismological Society of America. - 1996. — Vol. 86, Is. 4. P. 1091-1106.

Hariri-Ardebili M. A., Seyed-Kolbadi S. M., Saouma V. E., Salamon J., Rajagopalan B. Random finite element method for the seismic analysis of gravity dams // Engineering Structures. — 2018. Vol. 171. - P. 405-420.

Khokhlov N. I., Golubev V. I. On the Class of Compact Grid-Characteristic Schemes // Smart Modeling for Engineering Systems. - 2019. - P. 64-77. - Springer International Publishing.

Koketsu K., Fujiwaraand H., Ikegami Y. Finite-element Simulation of Seismic Ground Motion with a Voxel Mesh // Pure and Applied Geophysics. - 2004. - Vol. 161. - P. 2463-2478. 
